\title{
Assessment of continuous neutrophil CD64 index measurement for diagnosing sepsis and predicting outcome in a Chinese pediatric intensive care unit: a prospective study
}

\author{
Wei Cui ${ }^{1,2}$, Yuanyuan $\mathrm{Xu}^{3}$, Hui Fang ${ }^{2}$, Wenjia Tong ${ }^{3}$, Liran Zhu ${ }^{2}$, Danqun Jin ${ }^{3}$, Haipeng Liu ${ }^{1,2}$ \\ ${ }^{1}$ Department of Scientific Research and education, Anhui Provincial Children's Hospital (Children's Hospital of Anhui Medical University), Hefei, \\ China; ${ }^{2}$ Anhui Institute of Pediatric Research, Hefei, China; ${ }^{3}$ Pediatric Intensive Care Unit, Anhui Provincial Children's Hospital (Children's \\ Hospital of Anhui Medical University), Hefei, China \\ Contributions: (I) Conception and design: W Cui, H Fang, D Jin; (II) Administrative support: H Liu; (III) Provision of study materials or patients: Y \\ Xu, W Tong, D Jin; (IV) Collection and assembly of data: W Cui, Y Xu, W Tong, F Hui, L Zhu, D Jin; (V) Data analysis and interpretation: W Cui, \\ Y Xu, W Tong, F Hui, L Zhu, D Jin; (VI) Manuscript writing: All authors; (VII) Final approval of manuscript: All authors. \\ Correspondence to: Haipeng Liu. Department of Scientific Research and Education, Anhui Provincial Children's Hospital (Children's Hospital of \\ Anhui Medical University), No. 39, Wangjiang East Road, Baohe District, Hefei 230051, China. Email: forliterature@163.com.
}

\begin{abstract}
Background: The high affinity immunoglobulin-Fc fragment receptor I CD64 on neutrophils is widely assumed to be a useful biomarker in the early identification of sepsis, and it improves outcomes. We aimed to determine its ability to diagnose sepsis and predict its prognosis with continuous measurements.

Methods: A total of 335 patients admitted to a Chinese PICU were prospectively stratified into two groups according to the presence of sepsis (defined by clinical criteria for sepsis) between 2018 and 2019. Serum concentrations of the nCD64 index, C-reactive protein (CRP), and procalcitonin (PCT) were measured. Sensitivity, specificity and receiver operating characteristic (ROC) curves were calculated to evaluate the diagnostic value for sepsis. A multiple logistic regression model was used to estimate the prognostic value of continuous nCD64 index measurement for in-hospital death.

Results: The baseline nCD64 index and levels of PCT and CRP were significantly higher in septic children than in nonseptic children $(\mathrm{P}<0.05)$. The nCD64 index presented a higher sensitivity $(0.90)$, specificity $(0.78)$ and area under the ROC curve $[0.91(0.90,0.93)]$ than CRP and PCT in discriminating septic children with an optimal cutoff value of 5.78. The nCD64 index decreased with the progression of sepsis, and the baseline nCD64 index was strongly associated with in-hospital death (OR: 2.18, 95\% CI: 1.02-4.74). Moreover, the more rapidly the nCD64 index declined, the lower the in-hospital death rate was (OR: 0.89, 95\% CI: 0.63-1.35) after adjusting for the baseline nCD64 index and other confounders.

Conclusions: The nCD64 index was not only effective for the early diagnosis of childhood sepsis but also positively associated with the prognosis of sepsis. Moreover, the nCD64 decline was inversely associated with the in-hospital death rate.
\end{abstract}

Keywords: Sepsis; childhood; neutrophil CD64 index; diagnosis; outcome

Submitted Feb 10, 2021. Accepted for publication Apr 20, 2021.

doi: $10.21037 / \mathrm{tp}-21-63$

View this article at: http://dx.doi.org/10.21037/tp-21-63

^ ORCID: Wei Cui, 0000-0002-4814-1959; Yuanyuan Xu, 0000-0002-4814-1959. 


\section{Introduction}

Sepsis is the response of the immune system to invasive infection, which is the main cause of morbidity and mortality in the intensive care unit (ICU) globally, and the disease burden is extremely high (1-3). Although early identification and appropriate immediate management of sepsis were reported to be associated with better prognosis and outcomes (4), early diagnosis of this syndrome remains challenging. Hundreds of studies have investigated possible promising early diagnostic biomarkers for sepsis (5-7). In fact, the early biomarkers of sepsis reported in the literature have exceeded 100 and continue to increase, but none were proven to be consistently useful in the early diagnosis of sepsis. Among them, the most commonly mentioned biomarkers were C-reactive protein (CRP), procalcitonin (PCT) and in particular, the neutrophil CD64 index (nCD64 index).

CD64 is also called high-affinity immunoglobulin Fc- $\gamma$ receptor I (8). The $\mathrm{nCD} 64$ index has been investigated for years as a biomarker of infection and sepsis, given its reported low baseline expression and quick increase after inflammation (9). Moreover, several authors have also reported its value as an indicator of sepsis severity, prognosis and outcomes. Dai et al. (10) performed the diagnostic value of the neutrophil CD64 index for neonatal sepsis in 7 studies, and the pooled sensitivity and specificity were 80\% (95\% CI: 69-88\%) and 83\% (95\% CI: 71-90\%), respectively. Thiriet et al. (11) performed a prospective analysis in adults in the ICU and showed that the nCD64 index was a possible diagnostic marker of sepsis with good sensitivity and specificity (78\% and 70\%, respectively). Moreover, the author performed repeated measurements of the nCD64 index, which showed that it may help improve the accuracy of the diagnosis of sepsis. El Shimi et al. (12) observed significantly higher baseline neutrophil CD64 levels in deceased patients than those in recovered neonates, which indicated its predictive value for disease outcomes. However, most studies have focused on populations of neonates and adults, and childhood sepsis has seldom been observed. Moreover, few studies have focused on the change in the nCD64 index during treatment and its influence on disease prognosis.

The aim of this paper was to assess the role of the nCD64 index in establishing the diagnosis criteria of childhood sepsis, comparing the sensitivity, specificity and AUC of PCT, CRP and nCD64 in the diagnosis of childhood sepsis. Moreover, continuous measurement of the $\mathrm{nCD} 64$ index was performed to observe the changes in the nCD64 index during treatment and to evaluate its effect in predicting sepsis outcomes.

We present the following article in accordance with the STROBE reporting checklist (available at http://dx.doi. org/10.21037/tp-21-63).

\section{Methods}

\section{Study design and population}

This prospective study was implemented on participants admitted to the PICU of Anhui Provincial Children's Hospital between 2018 and 2019. Patients who exhibited the clinical signs of sepsis at admission were enrolled as the sepsis group. The nonsepsis group included those with no symptoms or signs of infection. Two pediatricians independently classified the diagnosis as sepsis or no sepsis at the time of admission.

In the present study, sepsis was defined as systemic inflammatory response syndrome (SIRS) caused by suspected or confirmed infection. The diagnosis of SIRS was based on at least two clinical criteria: hyperthermia (rectal temperature $>38^{\circ} \mathrm{C}$ ) or hypothermia (rectal temperature $<36^{\circ} \mathrm{C}$ ), tachycardia, tachypnea, and white blood cell (WBC) count $<4 \times 10^{9} / \mathrm{L}$ or $>12 \times 10^{9} / \mathrm{L}$. Furthermore, a child with sepsis had damaged function of at least one organ, such as consciousness changes, hypoxemia, increased levels of serum lactic acid and a fast pulse.

We excluded patients who were neonates or those $>18$ years old. Patients with chromosomal abnormalities, congenital anomalies and/or surgical problems were also excluded from the study. The study was conducted in accordance with the Declaration of Helsinki (as revised in 2013). The study was approved by the Ethics Committee of Anhui Provincial Children's Hospital, Hefei, China (NO. EYLL-2017-29) and informed consent was taken from all individual participants.

\section{Neutrophil CD64 index}

The neutrophil CD64 index was evaluated by flow cytometry (Becton Dickinson, FACS Canto II, USA). Briefly, $100 \mu \mathrm{L}$ peripheral blood samples were mixed with $20 \mu \mathrm{L}$ fluorescein isothiocyanate (FITC)-labeled CD64 monoclonal antibody, followed by gentle vortexing and incubation in the dark for $15 \mathrm{~min}$. Then, $1 \mathrm{~mL}$ diluted hemolysin solution for flow cytometry was added to the 
blood samples. The samples were vortexed and incubated for $15 \mathrm{~min}$ in the dark, and then they were subjected to flow cytometry on a FACS Canto II platform (Becton Dickinson, USA) to evaluate the mean FITC intensity from neutrophils and lymphocytes; the ratio of the two was calculated as the nCD64 index. During hospitalization, we performed three nCD64 index measurements in the sepsis group. The first was performed within 12 hours after admission as baseline; the second was performed on day 3 after admission, and the third measurement was taken the day before discharge. For the nonsepsis group, the nCD64 index was only measured once within 12 hours after admission.

\section{Laboratory assays}

Peripheral blood samples were collected under complete aseptic conditions on ethylenediaminetetracetic acid (EDTA) $(1.2 \mathrm{mg} / \mathrm{mL})$ for blood cell count and immunophenotyping. WBC count was performed on an automatic blood cell Coulter Sysmex XN-350 (Beckman Coulter, Inc., Fullerton, CA). Serum was obtained from clotted samples by centrifugation at $1,000 \times \mathrm{g}$ for $15 \mathrm{~min}$ and used for biochemical analysis of CRP and PCT. High sensitivity CRP and PCT levels were analyzed on an automatic biochemical analyzer (7600-020, Hitachi, Japan) with a turbidimetric CRP assay kit (Fuji, Japan) and an automatic electrochemiluminescence immunoanalyzer (Cobas E411, Roche Diagnostics, Germany), respectively.

\section{Statistical analysis}

Participant characteristics were stratified into different groups and presented as the mean [standard deviation (SD)] or median [interquartile range (IR)] for continuous variables and frequency (proportion) for categorical variables. The differences in population characteristics were compared using a $t$-test, the Wilcoxon-Mann-Whitney test, or the chi-square test.

Receiver operating characteristic (ROC) curves were generated to evaluate the diagnostic value of these biomarkers, and Youden's index was calculated to determine the optimal cutoff values of the biomarkers, together with the area under the ROC curve (AUC, with 95\% CI), sensitivity, specificity, positive and negative likelihood ratios and predictive values. Generalized linear models with a logit link were used to evaluate the predictive value of the baseline nCD64 index and its change from admission for in-hospital death rates with crude or full models adjusted for age, sex, sepsis group, pediatric critical illness score (PCIS) and other biomarkers.

A two-tailed $\mathrm{P}$ value $<0.05$ was considered to be statistically significant in all analyses. All statistical analyses were performed using R software (http://www.R-project.org).

\section{Results}

\section{Study participants and baseline characteristics}

Within a two-year period, a total of 335 participants were involved in the final analysis (Figure 1). Participants were divided into two groups (the sepsis group and nonsepsis group) according to the presence of sepsis. In this population, $120(35.0 \%)$ patients were classified into the sepsis group by two independent pediatric physicians according to clinical sepsis diagnostic criteria. The sepsis group had lower PCIS scores and higher baseline concentrations of the nCD64 index. Moreover, PCT, CRP and WBC counts were also positively higher in the sepsis group than in the nonsepsis group. The total in-hospital mortality was $2.4 \%$ in this study, with $4.2 \%$ and $1.4 \%$ in the sepsis group and nonsepsis group, respectively. Other general characteristics of the study participants are presented by sepsis status in Table 1. No significant differences were observed in age, sex or other variables.

\section{Clinical performance of biomarkers in the diagnosis of sepsis}

Baseline serum concentrations of the nCD64 index, CRP, PCT, and the WBC count were determined to assess their diagnostic value for sepsis. Overall, all four biomarkers were positively higher in the sepsis group $(\mathrm{P}<0.05)$. Areas under the ROC curves (AUCs) differed among these four biomarkers, with CD64 index at $0.91(0.90,0.93)$, PCT at $0.79(0.77,0.81)$, CRP at $0.68(0.66,0.71)$, and WBC count at $0.60(0.57,0.63)$. The nCD64 index had the highest AUC value, which showed the best diagnostic value for sepsis among these biomarkers (Figure 2).

The nCD64 index presented a sensitivity of 0.90 and a specificity of 0.78 with a cutoff value of 5.78 . The positive and negative likelihood ratios were 4.08 and 0.13 , respectively, and the positive and negative predictive values were $70 \%$ and $93 \%$, respectively. We observed that the other three biomarkers did not perform better than the nCD64 index (Table 2).

We also performed a multiple logistic regression model 


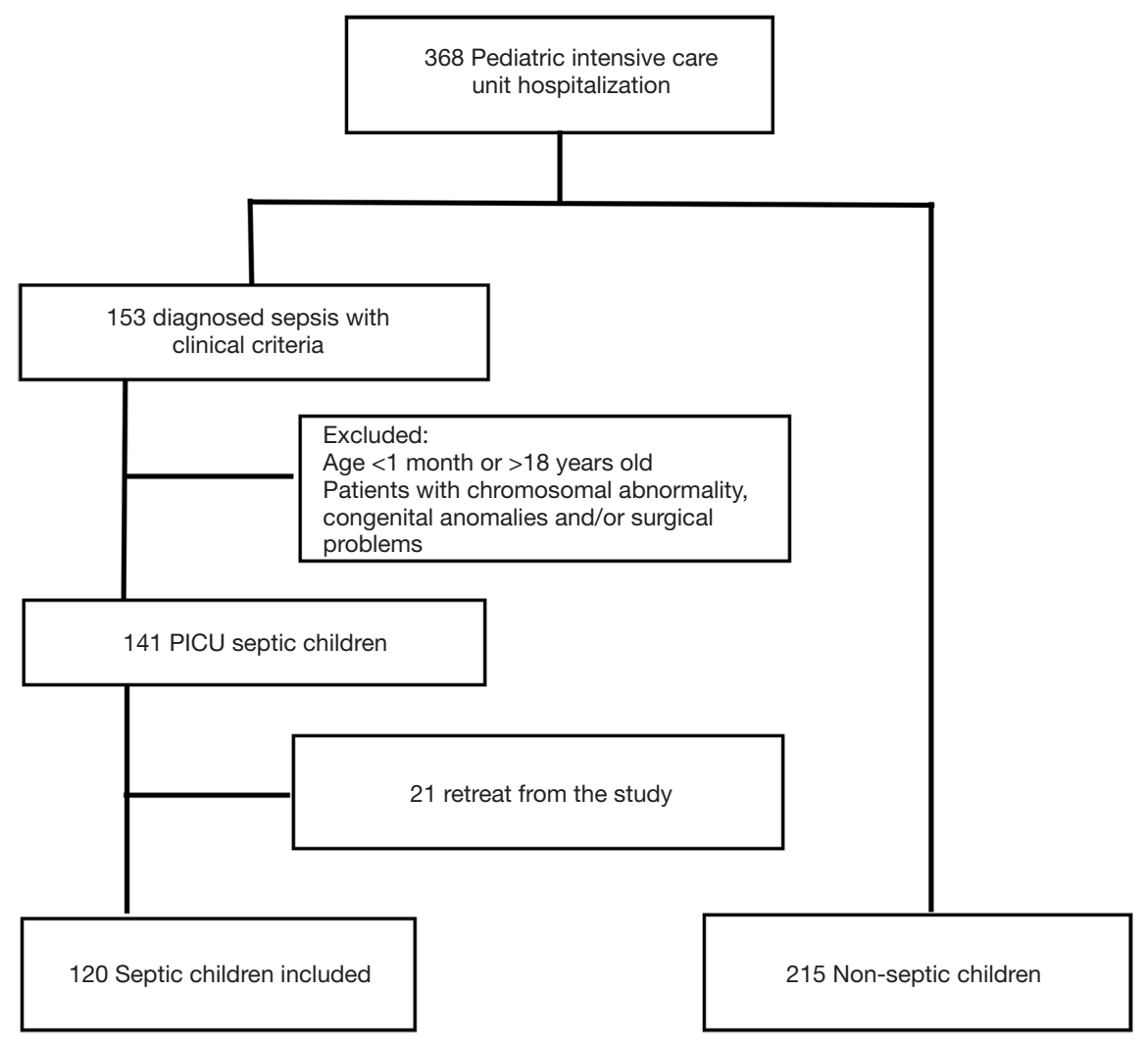

Figure 1 Diagram of participants included in the study.

Table 1 Baseline characteristics of two groups at admission

\begin{tabular}{|c|c|c|c|c|}
\hline & Non-sepsis $(n=215)$ & Sepsis $(n=120)$ & Total $(n=335)$ & $P$ value \\
\hline Age, yrs & $1.37(0.62,3.44)$ & $1.23(0.52,3.21)$ & $1.3(0.58,3.34)$ & 0.852 \\
\hline Boys & $119(55.3)$ & $72(60.1)$ & $191(57.0)$ & 0.436 \\
\hline Death & $3(1.4)$ & $5(4.2)$ & $8(2.4)$ & 0.223 \\
\hline LOS, days & $6(4.5,8)$ & $6[5,8]$ & $6[5,8]$ & 0.114 \\
\hline Baseline nCD64 index & $4.09 \pm 2.15$ & $6.06 \pm 2.14$ & $4.8 \pm 2.35$ & $<0.001$ \\
\hline CRP, mg/L & $11(6.12,24.3)$ & $20.9(12.3,29.6)$ & $14.8(8.56,26.7)$ & $<0.001$ \\
\hline PCT, ng/mL & $0.33(0.25,0.42)$ & $0.49(0.41,0.58)$ & $0.33(0.25,0.42)$ & $<0.001$ \\
\hline WBC, $10^{9} / \mathrm{L}$ & $9.16(6.38,14)$ & $11.31(8.2,16)$ & $9.21(6.34,14)$ & $<0.001$ \\
\hline
\end{tabular}

Results are presented as $\mathrm{n}(\%)$, mean (SD), or median (interquartile range). LOS, length of stay; PCIS, pediatric critical illness Score; SD, standard deviation; CRP, C-reactive protein; PCT, procalcitonin; WBC, white blood cell count.

to evaluate the predictive value of these biomarkers for the identification of sepsis. We observed a significantly increased risk of sepsis with an increased nCD64 index (OR:
2.35, 95\% CI: 2.15-2.60). Moreover, we also observed a significant effect of PCT (OR: 2.18, 95\% CI: 1.44-3.17), CRP (OR: 1.05, 95\% CI: 1.03-1.07) and WBC count (OR: 


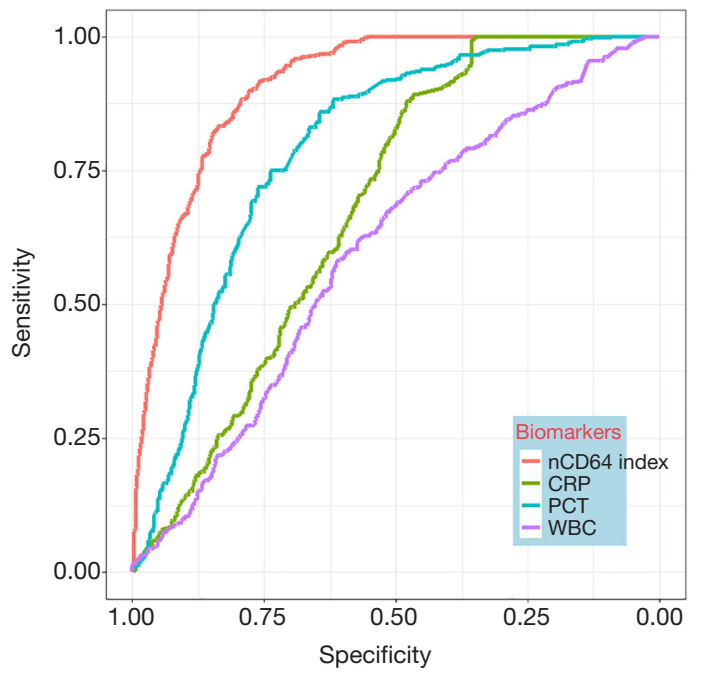

Figure 2 Receiver Operating Characteristic (ROC) curves of different biomarkers.
1.03, 95\% CI: 1.00-1.05) for sepsis prediction, but with lower odds ratio (Table 3 ).

\section{The performance of continuous CD64 index determination for death}

In the present study, significant differences were observed between the baseline nCD64 index and those assessed afterwards in the sepsis group $(\mathrm{P}<0.05)$. Moreover, a gradual decline in the nCD64 index was observed during treatment (Figure 3).

We also performed multiple logistic regression to evaluate the risk factors for in-hospital death. Overall, there was a significant association between nCD64 index concentrations and in-hospital death. We observed that the increase in the baseline nCD64 index was associated with a $118 \%$ increase in the adjusted risk of death (OR: 2.18, 95\%

Table 2 Comparison of clinical performance of biomarkers in diagnosing sepsis

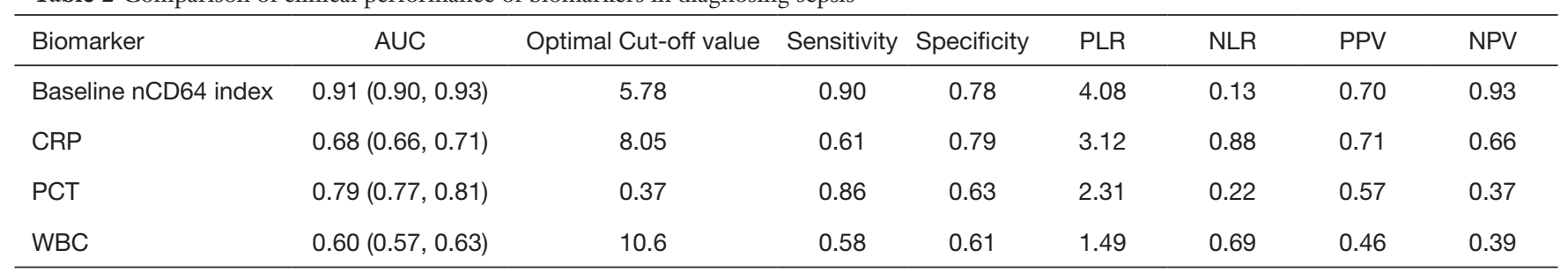

CRP, C-reactive protein; PCT, procalcitonin; WBC, white blood cell count; PLR, positive likelihood ratio; NLR, negative likelihood ratio; PPV, positive predictive value; NPV, negative predictive value.

Table 3 Multiple-logistic regression of these biomarkers for differentiating sepsis and non-sepsis children.

\begin{tabular}{lcc}
\hline \multirow{2}{*}{ Variables } & Crude & Adjusted $^{*}$ \\
\cline { 2 - 3 } Baseline nCD64 index & OR $(95 \% \mathrm{Cl})$ & OR (95\% Cl) \\
CRP & $2.38(2.18,2.62)$ & $2.35(2.15,2.60)$ \\
PCT & $1.05(1.04,1.06)$ & $1.05(1.03,1.07)$ \\
WBC & $1.21(0.92,1.59)$ & $2.18(1.44,3.17)$ \\
Second nCD64 index & $0.99(0.98,1.101)$ & $1.03(1.00,1.05)$ \\
Third nCD64 index & $1.19(1.12,1.27)$ & $1.19(1.12,1.28)$ \\
\hline
\end{tabular}

*, Adjusted for: age, gender, PCIS score and these biomarkers. CRP, C-reactive protein; PCT, procalcitonin; WBC, white blood cell count; OR, odds ratio. 
CI: 1.02-4.74). We also observed an increasing trend of the second (OR: 1.29, 95\% CI: 0.93-1.65) and third nCD64 index measurements (OR: 1.12, 95\% CI: 0.74-1.54) with in-hospital death, although a nonsignificant association was observed.

To evaluate the association between the decrease in CD64 index during hospitalization and outcomes, we defined dCD64 as the baseline nCD64 index minus the third nCD64 index measurement. In the crude model, the increase in dCD64 was significantly associated with increased in-hospital death (OR: 1.31, 95\% CI: 1.07-1.58). However, after adjustments for the baseline CD64 index

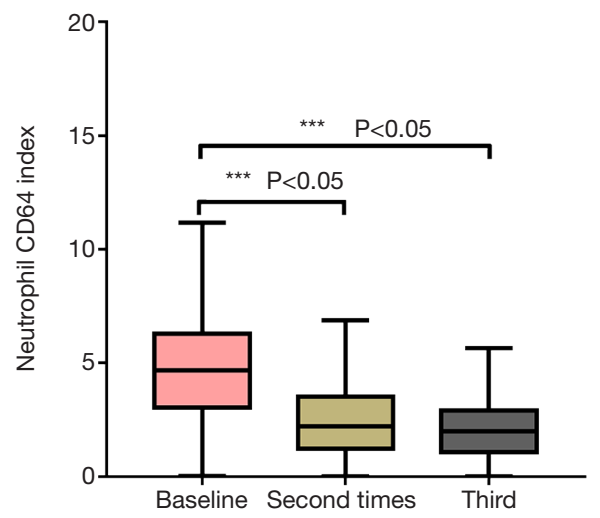

Figure 3 Evolution of continuous neutrophil CD64 index measurement among sepsis patients. and other confounders, the associations reversed (OR: 0.89, 95\% CI: $0.63-1.35$ ), even though the $\mathrm{P}$ value was not significant (Table 4).

\section{Discussion}

To our knowledge, this is the first prospective analysis in children to evaluate the early diagnostic value of nCD64 in sepsis and to examine the predictive value of continuous nCD64 index measurement for in-hospital death. We found that $\mathrm{nCD} 64$ had a better diagnostic value for sepsis than PCT, CRP and WBC count. We also determined that the baseline nCD64 index was associated with higher inhospital mortality. Moreover, the more rapid the decline in the $\mathrm{nCD} 64$ index during treatment was, the lower the risk of in-hospital death.

The treatment of sepsis is a dilemma without a definite early diagnosis. On the one hand, the overuse of antibiotics may lead to antimicrobial resistance and higher healthcare costs; on the other hand, it may aggravate the disease and increase mortality without timely treatment. Thus, the early diagnosis of sepsis is extremely important in clinical practice. In fact, the early diagnostic value of the nCD64 index for sepsis has been demonstrated in many studies focusing on elderly adults and neonates with promising results (13), but few studies have reported this topic in childhood (9). For example, Dai et al. (10) performed a meta-analysis including 7 studies in neonates with pooled

Table 4 Risk factors of hospital death rate

\begin{tabular}{lcc}
\hline \multirow{2}{*}{ Variables } & Crude & Adjusted $^{*}$ \\
\cline { 2 - 3 } Age & OR $(95 \%$ Cl $)$ & $0.93(0.60,1.26)$ \\
Boys & $1.02(0.74,1.29)$ & $1.31(0.28,5.92)$ \\
PCIS Score & $1.34(0.32,5.70)$ & $1.36(0.76,2.29)$ \\
Baseline nCD64 index & $0.81(0.68,0.98)$ & $2.18(1.02,4.74)$ \\
Second nCD64 index & $1.39(1.15,1.68)$ & $1.31(0.93,1.72)$ \\
Third nCD64 index & $1.29(0.93,1.65)$ & $1.13(0.74,4.60)$ \\
CRP & $1.12(0.74,1.54)$ & $0.99(0.91,1.06)$ \\
PCT & $1.01(0.95,1.07)$ & $0.26(0.00,2.44)$ \\
WBC & $0.83(0.03,2.67)$ & $1.11(1.00,1.24)$ \\
dCD64 index & $1.08(0.97,1.18)$ & $0.89(0.63,1.35)$ \\
\hline
\end{tabular}

*, adjusted for: age, gender, PCIS score and other biomarkers. dCD64 index, Baseline nCD64 index- Third nCD64 index. CRP, C-reactive protein; PCT, procalcitonin; WBC, white blood cell count; OR, odds ratio. 
sensitivity and specificity of $80 \%$ (95\% CI: $69-88 \%$ ) and 83\% (95\% CI: 71-90\%), respectively. Ye et al. (14) reported a sensitivity of $83 \%$ and specificity of $88 \%$ in diagnosing sepsis in 62 adult ICU patients. Groselj-Grenc et al. (15) reported the diagnostic performance of the nCD64 index with a sensitivity of $86 \%$ and specificity of $100 \%$ in children. In the present study, we evaluated the early diagnostic value of the nCD64 index with a sensitivity of 0.90 and specificity of 0.78 with an optimal cutoff value of 5.78 , which showed better effectiveness than PCT, CRP and WBC count with the highest AUC of $0.91(0.90,0.93)$ and was consistent with the results of a previous meta-analysis. In this study, we observed an optimal cutoff value of 5.78 , which is higher than that in neonates (16-18). These discrepancies may be explained by differences in methodology, time of sampling, and age of participants.

Thiriet et al. (11) reported a repeated measurement of the nCD64 index in adults and correctly classified $85 \%$ of sepsis cases on day 2 versus $74 \%$ on day 1 , which showed that repeated measurements may help improve the accuracy of sepsis diagnosis. In the present study, continuous measurement of the nCD64 index was performed over the course of disease, and a gradual decline in the nCD64 index was observed throughout treatment, which indicated that the nCD64 index can be used as a potential indicator for evaluating treatment effectiveness and progression. The result was consistent with the result in adults (19). We also determined the low diagnostic value of the second and third nCD64 indices with sensitivities of $77 \%$ and $54 \%$, respectively, which may be explained by the timely use of antibiotics.

We also evaluated the performance of the continuous nCD64 index in predicting death, and a significant association between the baseline nCD64 index and inhospital death was observed (OR: 2.18, 95\% CI: 1.02-4.74), which is consistent with previous studies in neonates $(12,20)$. In this study, a positive significant association between nCD64 index decline and death was observed (OR: 1.31, 95\% CI: $1.07-1.58)$ in the crude model. Interestingly, after adjustments of the baseline nCD64 index and other confounders, the associations reversed (OR: 0.89, 95\% CI: $0.63-1.35)$, even though the $\mathrm{P}$ value was not significant. The inverse result not only revealed the core value of the baseline nCD64 index in predicting death but also indicated that the nCD64 index decline was negatively associated with in-hospital death rates.

In the present study, although the potential value of early discrimination and prediction of death for sepsis of nCD64 index were observed, its application in some primary hospital may be limited due to the use of flow cytometer is required. There were two alternatives for this situation. Firstly, the correlation between the nCD64 index and other markers (WBC, PCT and CRP) were calculated to evaluate its possibility to be able to instead of nCD64. In this study, although these biomarkers were inferior to nCD64 index in early diagnosing sepsis and predicting hospital death, its potential value were observed; we also calculated the correlation coefficient between these indicators and nCD64 index and found these markers were positively correlated with nCD64 index (WBC: 0.31; PCT: 0.32; CRP: 0.28) and could be used as a substitute for nCD64 index. Secondly, with the development of microfluidic affinity cells separation system, the simple and multiparameter combined microfluidic chip for early diagnosis of sepsis were exploited, including culture-negative sepsis and culture-positive sepsis, and excellent diagnosis performance were observed (and AUC value of single CD64 index was 0.90 and the combination of CD64 and CD69 for sepsis diagnosis had the AUC of 0.98) (21-23).

Several potential concerns or limitations are worth mentioning. First, the diagnosis of sepsis was based on clinical criteria rather than blood culture tests in this study. In fact, some studies have demonstrated the difference in the nCD64 index between culture-positive sepsis patients and clinical sepsis patients with negative cultures, and no significant difference was observed $(24,25)$. Second, our current study defined in-hospital death as the study outcome and did not conduct further follow-up after discharge; thus, it is possibly unable to demonstrate the long-term effect of sepsis. Third, we calculated the PCIS score rather than the acute physiology and chronic health evaluation (APACHE) score and Sepsis-related Organ Failure Assessment (SOFA) score in the current study, as the PCIS score is generally acknowledged as an excellent tool in China for judging the severity of childhood diseases and predicting the risk of death. Finally, our study was conducted in Chinese children. Whether the results can be extrapolated to other populations requires further verification. Due to these limitations, further confirmation is greatly needed.

\section{Conclusions}

In this prospective analysis of Chinese children with sepsis, the nCD64 index could be helpful for the early discrimination of childhood sepsis. We observed a significant association between baseline $\mathrm{nCD} 64$ index concentrations and in-hospital death. Moreover, an inverse 
association was observed between nCD64 index decline and in-hospital death rates.

\section{Acknowledgments}

We express our deep gratitude to the patients and the families for their willingness and cooperation in the study. Funding: This work was supported by the Key Research and Development Program Projects in Anhui, China (ID: 1704a0802172).

\section{Footnote}

Reporting Checklist: The authors have completed the STROBE reporting checklist. Available at http://dx.doi. org/10.21037/tp-21-63

Data Sharing Statement: Available at http://dx.doi. org/10.21037/tp-21-63

Conflicts of Interest: All authors have completed the ICMJE uniform disclosure form (available at http://dx.doi. org/10.21037/tp-21-63). The authors have no conflicts of interest to declare.

Ethical Statement: The authors are accountable for all aspects of the work in ensuring that questions related to the accuracy or integrity of any part of the work are appropriately investigated and resolved. The study was conducted in accordance with the Declaration of Helsinki (as revised in 2013). The study was approved by the Ethics Committee of Anhui Provincial Children's Hospital, Hefei, China (NO. EYLL-2017-29) and informed consent was taken from all individual participants.

Open Access Statement: This is an Open Access article distributed in accordance with the Creative Commons Attribution-NonCommercial-NoDerivs 4.0 International License (CC BY-NC-ND 4.0), which permits the noncommercial replication and distribution of the article with the strict proviso that no changes or edits are made and the original work is properly cited (including links to both the formal publication through the relevant DOI and the license). See: https://creativecommons.org/licenses/by-nc-nd/4.0/.

\section{References}

1. Liu V, Escobar GJ, Greene JD, et al. Hospital deaths in patients with sepsis from 2 independent cohorts. JAMA 2014;312:90-2.

2. Hershey TB, Kahn JM. State Sepsis Mandates - A New Era for Regulation of Hospital Quality. N Engl J Med 2017;376:2311-3.

3. Singer M, Deutschman CS, Seymour CW, et al. The Third International Consensus Definitions for Sepsis and Septic Shock (Sepsis-3). JAMA 2016;315:801-10.

4. Xini A, Pistiki A, Lada M, et al. Association of the early absolute CD64-expressing neutrophil count and sepsis outcome. Eur J Clin Microbiol Infect Dis 2019;38:1123-8.

5. Miyake F, Ishii M, Hoshina T, et al. Analysis of the Physiological Variation in Neutrophil CD64 Expression during the Early Neonatal Period. Am J Perinatol 2016;33:1377-81.

6. Sandquist M, Wong HR. Biomarkers of sepsis and their potential value in diagnosis, prognosis and treatment. Expert Rev Clin Immunol 2014;10:1349-56.

7. Elawady S, Botros SK, Sorour AE, et al. Neutrophil CD64 as a diagnostic marker of sepsis in neonates. J Investig Med 2014;62:644-9.

8. Hassan U, Ghonge T, Reddy B, Jr., et al. A point-of-care microfluidic biochip for quantification of CD64 expression from whole blood for sepsis stratification. Nat Commun 2017;8:15949.

9. Hoffmann JJ. Neutrophil CD64 as a sepsis biomarker. Biochem Med (Zagreb) 2011;21:282-90.

10. Dai J, Jiang W, Min Z, et al. Neutrophil CD64 as a diagnostic marker for neonatal sepsis: Meta-analysis. Adv Clin Exp Med 2017;26:327-32.

11. Thiriet C, Mahjoub K, Courte G, et al. Automated measurement of neutrophil CD64 expression for diagnosing sepsis in critically ill patients. Minerva Anestesiol 2019;85:943-50.

12. El Shimi MS, Abou Shady NM, Hamed GM, et al. Significance of neutrophilic CD64 as an early marker for detection of neonatal sepsis and prediction of disease outcome. J Matern Fetal Neonatal Med 2017;30:1709-14.

13. Farias MG, de Lucena NP, Dal Bo S, et al. Neutrophil CD64 expression as an important diagnostic marker of infection and sepsis in hospital patients. J Immunol Methods 2014;414:65-8.

14. Ye Z, Zou H, Liu S, et al. Diagnostic performance of neutrophil CD64 index in patients with sepsis in the intensive care unit. J Int Med Res 2019;47:4304-11.

15. Groselj-Grenc M, Ihan A, Derganc M. Neutrophil and monocyte CD64 and CD163 expression in critically ill neonates and children with sepsis: comparison of 
fluorescence intensities and calculated indexes. Mediators Inflamm 2008;2008:202646.

16. Du J, Li L, Dou Y, et al. Diagnostic utility of neutrophil CD64 as a marker for early-onset sepsis in preterm neonates. PLoS One 2014;9:e102647.

17. Lam HS, Wong SP, Cheung HM, et al. Early diagnosis of intra-abdominal inflammation and sepsis by neutrophil CD64 expression in newborns. Neonatology 2011;99:118-24.

18. Lam HS, Cheung HM, Poon TC, et al. Neutrophil CD64 for daily surveillance of systemic infection and necrotizing enterocolitis in preterm infants. Clin Chem 2013;59:1753-60.

19. Dimoula A, Pradier O, Kassengera Z, et al. Serial determinations of neutrophil CD64 expression for the diagnosis and monitoring of sepsis in critically ill patients. Clin Infect Dis 2014;58:820-9.

20. Hanna MOF, Abdelhameed AM, Abou-Elalla AA, et al. Neutrophil and monocyte receptor expression in patients with sepsis: implications for diagnosis and $\mathrm{p}$ rognosis of

Cite this article as: Cui W, Xu Y, Fang H, Tong W, Zhu L, Jin D, Liu H. Assessment of continuous neutrophil CD64 index measurement for diagnosing sepsis and predicting outcome in a Chinese pediatric intensive care unit: a prospective study. Transl Pediatr 2021;10(6):1668-1676. doi: 10.21037/tp-21-63 sepsis. Pathog Dis 2019;77:ftz055.

21. Zhang Y, Li W, Zhou Y, et al. Detection of sepsis in patient blood samples using CD64 expression in a microfluidic cell separation device. Analyst 2017;143:241-9.

22. Zhang Y, Zhou Y, Li W, et al. Multiparameter Affinity Microchip for Early Sepsis Diagnosis Based on CD64 and CD69 Expression and Cell Capture. Anal Chem 2018;90:7204-11.

23. Zhou Y, Zhang Y, Johnson A, et al. Combined CD25, CD64, and CD69 biomarker panel for flow cytometry diagnosis of sepsis. Talanta 2019;191:216-21.

24. Abdel-Aleem NF, Sorour AS, Elkholy YS, et al. Diagnostic Role of CD64 on Different Immune Cells in Early Diagnosis of Neonatal Sepsis. Egypt J Immunol 2018;25:35-44.

25. Zhou Y, Zhang Y, Johnson A, et al. Detection of culture-negative sepsis in clinical blood samples using a microfluidic assay for combined CD64 and CD69 cell capture. Anal Chim Acta 2019;1062:110-7. 